\title{
Simultaneous genotyping of multiple polymorphisms in human serotonin transporter gene and detection of novel allelic variants
}

\author{
R Avula, A Rand, JL Black and DJ O'Kane
}

The serotonin transporter, called SLC6A4, SERT or 5-HTT, modulates neurotransmission by removal of serotonin from the synapse of serotonergic neurons, facilitating serotonin reuptake into the presynaptic terminus. Selective serotonin reuptake inhibitors block the action of the serotonin transporter and are used to treat depression and other neuropsychiatric disorders. Three polymorphisms in the 5-HTT gene have been implicated in treatment response and neuropsychiatric disorders. A 44-bp promoter ins/del polymorphism (5-HTTLPR) produces primarily long and/or short alleles due to either 14 (short) or 16 (long) repeats of variably conserved 20-23 bp units. Also implicated, a 17-18 bp variable number tandem repeat found in intron2 (StIn2) is expressed as triallelic content with 9, 10, or 12 repeats (StIn2.9, StIn2.10 or StIn2.12). Finally, a single nucleotide polymorphism rs25531 located within the promoter polymorphic-linked region alters the function of the long promoter allele. We developed a PCR-based fragment analysis assay, which is analyzed on an ABI sequencer, whereby we are able to detect all three genotypes simultaneously. Using this technique, we identified novel sequences, which demonstrate promoter repeat regions containing (1) a 17 repeat with rs25531 A/G polymorphism, (2) two with 18-repeat units, (3) one with 20-repeat units and (4) a 24-repeat sequence. The novel repeats were confirmed by direct sequencing of gel-purified amplicons.

Translational Psychiatry (2011) 1, e32; doi:10.1038/tp.2011.34; published online 16 August 2011

\section{Introduction}

The serotonin transporter in the brain is the principal site of action for many antidepressant medications in treating major depressive disorders. The serotonin transporter gene (5-HTT) has several polymorphic loci that affect its expression or function. The promoter polymorphism known as the 5-HTTlinked polymorphic region (5-HTTLPR) has an ins/del in the 5-HTTLPR and most commonly is composed of a long allele $(\mathrm{L})$ or short allele $(\mathrm{S})$ (14 or 16 repeats of the $20-23 \mathrm{bp}$ unit). Variations in the promoter region of $5-H T T$ have been reported to be associated with differential remission rates following selective serotonin reuptake inhibitor medication treatment. ${ }^{1,2}$ The long form of the ins/del promoter variant has 43 or 44 more nucleotides than the short form and has been shown to drive transcription levels to more than twice the level of the $\mathrm{S}$ allele. ${ }^{3}$ In some previous studies, European subjects homozygous for the $L$ allele of the ins/del promoter variant have been reported to benefit more from treatment with medications that block serotonin reuptake than subjects without the L allele. ${ }^{1,4}$ Nakamura et al., ${ }^{5}$ after isolating and sequencing all the repeat units in the promoter, categorized four kinds of $S$ allelic variants and six different $L$ allelic variants, based upon the sequence of the different repeats. The $S$ alleles were 14-A, 14-B, 14-C, 14-D, and the $L$ alleles were $16-A, 16-B, 16-C, 16-D, 16-E$ and $16-F$. They also observed longer repeats composed of 19,20 and 22 units; extra long versions of the LPR with 18-, 19- and 20-repeat units were also reported by others. ${ }^{6-8}$ Recently a 17 -repeat allele and an extra short allele of 11 repeats was reported. ${ }^{8}$ Hu et al. ${ }^{3}$ designated the $L$ allele with an adenosine at single nucleotide polymorphism (SNP) rs25531 as $L_{A}$ and the $L$ allele with a guanine at rs25531 as $L_{G}$. The $L_{A}$ and $L_{G}$ designation corresponds to alleles $16-\mathrm{A}$ and $16-\mathrm{D}$, respectively, in Nakamura's nomenclature. The $L_{A}$ was reported to have higher activity than $\mathrm{L}_{\mathrm{G}}{ }^{3}$. The $\mathrm{A} \rightarrow \mathrm{G}$ substitution is in the $\mu$ unit (sixth unit) and this gave rise to six different genotypes in the HTTLPR-SS, $L_{G} L_{G}, S L_{G}, L_{A} L_{G}, S L_{A}, L_{A} L_{A}$, where $S S$ expressed the least HTT mRNA and $L_{A} L_{A}$ expressed the most, and the two heterozygous genotypes that contain one copy of the $S$ allele did not exhibit lower than expected expression as predicted by the $\mathrm{S}$-dominant model. ${ }^{3}$ The $\mathrm{L}_{\mathrm{G}}$ allele created a stronger AP2-DNA binding site which, in turn, suppressed transcription. The $L_{A}$ allele was also associated with higher 5-HTT-binding potential, an index for 5-HTT density in putamen using 3-(11) C-amino-4-(2-dimethylaminomethylphenyl-sulfanyl) benzonitrile ( $\left.\left[{ }^{11} \mathrm{C}\right] \mathrm{DASB}\right)$ positron emission tomography. ${ }^{9}$ The $G$ substitution is also observed in the context of $S$ allele $\left(S_{G}\right)$, which is very rare and was designated as $14-\mathrm{B}$ and $14-\mathrm{D}$ by Nakamura. ${ }^{5}$ The finding that rs25531 is also associated with selective serotonin reuptake inhibitor response demand that both 5-HTTLPR and rs25531 be viewed as two independent loci for genotyping, and demonstrate a need for more comprehensive genotyping procedures. ${ }^{10}$

Department of Laboratory Medicine and Pathology, Nucleotide Polymorphism Lab, Mayo Clinic, Rochester, MN, USA

Correspondence: R Avula, Nucleotide Polymorphism Lab, Department of Laboratory Medicine and Pathology, Institution Mayo Clinic, Hilton 400, 200 1st street SW, Rochester, MN 55905, USA.

E-mail: avula.rajeswari@mayo.edu

Keywords: fragment analysis; 5-HTT; rs25531; serotonin transporter; VNTR

Received 26 April 2011; revised 21 June 2011; accepted 7 July 2011 
Studies have shown that the StIn2, another variable number tandem repeat (VNTR) region, may act as a transcriptional enhancer of the 5-HTT gene, with 12-repeat allele having more activity than the 10-repeat allele. ${ }^{11}$ The allele frequency of the 9 repeat is low (1-3\% in the European population). The intronic polymorphism has been mainly investigated in affective illness. Association of StIn2.9 with unipolar depression was shown by Ogilvie et al. ${ }^{12}$ StIn2.12 has been shown to be associated with bipolar disorder in multiple studies. ${ }^{6,13,14}$ Potential association/linkage disequilibrium of the intronic polymorphism and schizophrenia was also shown. ${ }^{15}$

Hranilovic et al. ${ }^{11}$ demonstrated the combined effect of the 5-HTTLPR and StIn2 polymorphism, they separated the groups into high-expressing genotypes at both loci ( $L / L ~ 12 / 12)$, low-expressing genotypes at one locus (L/L 10 and S 12/12) and those containing low-expressing genotypes (S 10). The mean 5-HTT expression was the highest with two highexpressing genotypes, $20 \%$ lower with one low-expressing genotypes and 50\% lower with low-expressing genotype at both the loci. Lovejoy et al. ${ }^{16}$ suggested that there is an additional layer of transcriptional complexity based on the primary sequence of the VNTR, and that the controversy as to the correlation of VNTR copy number with predisposition to affective disorders could be resolved by reanalyzing the data and taking into account the primary sequence of the VNTR.

The method we developed to genotype 5-HTTLPR, StIn2 and the SNP rs25531 uses fluorescently labeled PCR primers. An advantage of a fluorescent dye detection system is that DNA fragments overlapping in size range can be labeled with different dyes and thus be simultaneously detected in a single lane or by injection on electrophoresis instrumentation. Since both the 5-HTTLPR and StIn2 are composed of repeat regions, there is a possibility of size overlap. Labeling with two different dyes provides a significant increase in throughput over the more traditional methods in detecting the different alleles. Using alternative fluorescent dyes for the two separate loci, LPR and IN2, along with designing the products to yield defined and unique fragment length, enables the amplicons generated in the four separate PCRs to be separated for the four different reactions in the same well during electrophoresis. Furthermore the amplifications were designed to utilize the same cycling conditions to generate the PCR amplicons. The allele-specific PCR for the rs25531 eliminates the restriction enzyme digest of the PCR amplicon thereby further streamlining the assay and reducing the chance of errors while lowering the cost of performing the assay further. Here we present the simultaneous genotyping of multiple polymorphisms in human serotonin transporter gene and the novel variants identified.

\section{Materials and methods}

Generating the PCR fragments. Genomic DNA was extracted using the Qiagen EZ-1 extraction method (Hilden, Germany). The assay was composed of four separate amplifications as follows: amplify the ins/del region of the promoter using primers 5HTTLPRF and 6FAM5HTTLPRR, to amplify the StIn2 region with primers 5HTTIN2F and HEX5HTTIN2R, and two additional reactions to examine the SNP rs25531 region using two allele specific primers
Table 1 Primers and Primer sequences

\begin{tabular}{ll}
\hline Primers & Sequence $\left(5^{\prime}-\mathbf{3}^{\prime}\right)$ \\
\hline 5HTTLPRF & TGAATGCCAGCACCTAACCC \\
FAM5HTTLPRR & TTCTGGTGCCACCTAGACGC \\
5HTTIN2F & GGGGTCAGTATCACAGGCTGC \\
HEX5HTTIN2R & TCATGTTCCTAGTCTTACGCCAGTG \\
5HTTSNPAF & TTCACCCCTCGCGGCAT \\
& CCCCCCTGCACCCACA \\
5HTTSNPGF & CGCGGCATCCCCCCTGCACCCACG \\
5HTTM13(LPR)F & GGGTTCCCTAAGGGTTGGAG \\
& GCGTTGCCGCTCTGAATGC \\
5HTTM13(LPR)R & GTGCCAGCAAGATCCAATCTAGA \\
& GAGGGACTGAGCTGGACAACCAC \\
5HTTIN2M13F & GGGTTCCCTAAGGGTTGGAA \\
& ATGTCTGGCGCTTCCCCTAC \\
5HTTIN2M13R & GTGCCAGCAAGATCCAATCTA \\
& GAAAGCATCATGTTCCTAGTC \\
M13_seq_F & GGGTTCCCTAAGGGTTGGA \\
M13_seq_R & GTGCCAGCAAGATCCAATCTAGA \\
\hline
\end{tabular}

5HTTSNPAF and 5HTTSNPGF, with a common reverse primer 6FAM5HTTLPRR. The promoter region reverse primer and the StIn2 region reverse primer were fluorescently labeled with 6FAM and HEX, respectively. The primer sequences are given in Table 1. In a 12.5- $\mu$ l reaction volume, $25 \mathrm{ng}$ of genomic DNA were amplified with $2.5 \mu \mathrm{l}$ of $5 \times$ Promega Flexi buffer, $1 \mu \mathrm{l}$ of $25 \mathrm{mM} \mathrm{MgCl}_{2}, 0.5 \mu \mathrm{l}$ of $10 \mathrm{~mm}$ dNTP mix, $3 \mu \mathrm{l}$ of $5 \mathrm{M}$ betaine (Sigma-Aldrich, St Louis, MO, USA) and $0.1 \mu$ l of Promega GoTaq, Hot start polymerase (Promega Corporation, Madison, WI, USA). The primers were used at $25 \mu \mathrm{M}$ concentration: $0.5 \mu \mathrm{l}$ was used in the PCR reaction, except the allele specific primers, which were $0.1 \mu \mathrm{l}$ in the reaction. Thermal cycling consisted of $2 \mathrm{~min}$ of denaturation at $94^{\circ} \mathrm{C}$ followed by 30 cycles of $94{ }^{\circ} \mathrm{C}(30 \mathrm{~s})$, $62{ }^{\circ} \mathrm{C}(30 \mathrm{~s})$ and $72{ }^{\circ} \mathrm{C}(60 \mathrm{~s})$, and with a final extension step of $10 \mathrm{~min}$ at $72^{\circ} \mathrm{C}$.

Detection on $A B|3130 x|$ genetic analyzer. The PCR products were analyzed on $\mathrm{ABI} 3130 \mathrm{xl}$ Genetic analyzer. A volume of $1 \mu \mathrm{l}$ of sample (composed by pooling $2 \mu \mathrm{l}$ from each of the four amplicons) was added to a mix of $0.5 \mu \mathrm{GS}$ 600Liz size marker (Applied Biosystems, Foster City, CA, USA) and $9 \mu \mathrm{l}$ of Hi-Di formamide (Applied Biosystems). A volume of $9 \mu \mathrm{l}$ of the marker mix and $1 \mu \mathrm{l}$ of pooled amplicon was denatured at $95^{\circ} \mathrm{C}$ for $5 \mathrm{~min}$, cooled on an ice-water slurry for $5 \mathrm{~min}$, and subsequently run on the $\mathrm{ABI} 3130 \mathrm{xl}$ analyzer. The data were analyzed using GeneMarker v1.9 (SoftGenetics, State College, PA, USA).

Sequence confirmation. The samples analyzed by fragment analysis were verified by direct DNA sequencing. The primers used for generating the sequencing amplicons and sequencing primer details are given in Table 1. The primers used to generate the amplicons for sequencing have M13 sequences on the $5^{\prime}$ end. This simplifies the sequencing by using two common primers for any number of specific regions. Direct sequencing was performed using BigDye terminator v3.1/Sequencing standard kit (Applied Biosystems) following the manufacturer's directions and separating products on an ABI 3130xI. 
The sequence data were analyzed using MutationSurveyor (SoftGenetics). The samples heterozygous ( $L$ and $S$ ) for the promoter region were complex and difficult to analyze using the software. To confirm the results we obtained, the amplicons were separated on a $2 \%$ agarose gel, and the short and the long bands were excised and purified using GeneClean (Qbiogene, Solon, OH, USA) before sequencing. a

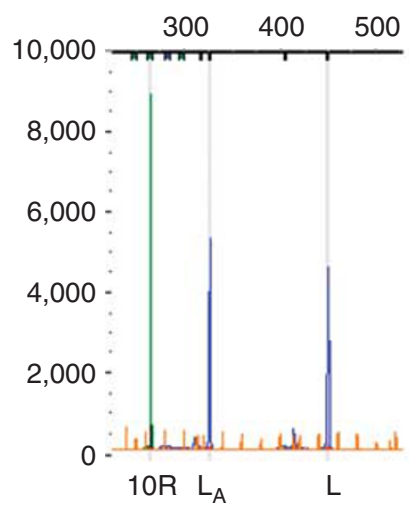

b

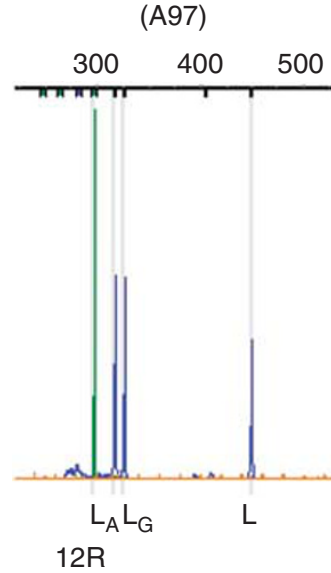

C

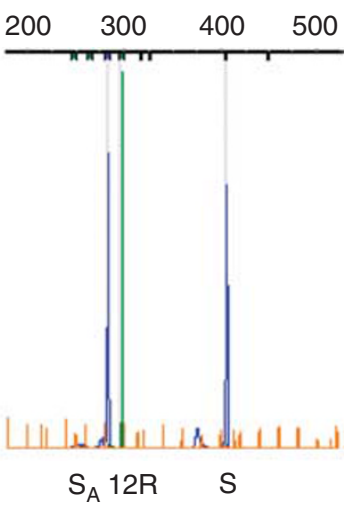

d

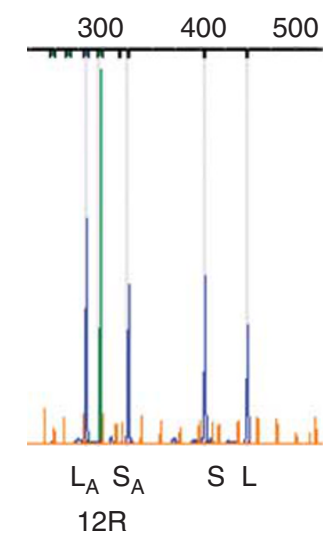

Figure 1 Electropherogram from $A B|3130 x|$. 1a was a $L_{A} / L_{A}$ for promoter and $S$ tln2.10. 1b was a $L_{A} / L_{G}$ and $S t \ln 2.12$. 1c was a $S_{A} / S_{A}$ and $S \ln 2.12$ and $1 \mathrm{~d}$ was a $S_{A} / L_{A}$ with $S t \ln 2.12$.

\section{Results}

Genotyping by fragment analysis. Since the reverse primers of the promoter region and the Stln2 region were fluorescently labeled, we were able to detect the alleles simultaneously by mixing the PCR products. The different alleles expected for the promoter region are $\mathrm{L}$ (long), $\mathrm{S}$ (short), $L_{A}, L_{G}, S_{A}$ and $S_{G}$. The Stln2 VNTR alleles would be StIn2.9, 10, and 12 (9R, 10R and $12 \mathrm{R}$ repeats). The expected peak sizes are shown in Figure 1 and Table 2 (R15, A97, M09 and C126 are DNA from the Coriell repository, Camden, NJ, USA). The $L$ allele peak was at 450 and the $S$ allele peak was at 406 . The $L_{A}$ peak was at 327 , and the $G$ allele-specific primers were made nine bases shorter than the $A$ allele-specific primer so the $L_{G}$ peak was at 318, while the $S_{A}$ peak was at 284 (the $S_{G}$ peak should be 275 bases but we did not find any $S_{G}$ peak in any of the samples reported here). The StIn2 alleles yielded fragments of 248 (9), 265 (10) and 298 (12). The software was set to allow a variation of \pm 3 for the promoter polymorphisms and \pm 3 for the StIn2. As the reverse primer for the promoter region (6FAM5HTTLPRR) was the same for the 5-HTTLPR and the allele-specific PCR, the change in the size of the L/S allele(s) found in the variant sequences was also reflected in the $A / G$ fragment.

Identification of novel alleles. We detected novel alleles using the genotyping method we developed. The peak sizes of the new alleles detected are shown in Figure 2 and Table 2, and the fragments longer than the $L$ allele were called extra long $(\mathrm{XL})$. Sample 1 was a heterozygous sample for the 5-HTTLPR polymorphism with $S$ and $S_{A}$ peaks and an $\mathrm{XL}$ peak and StIn2.9/12 genotype for StIn2. The XL peak was at 470 instead of 450 , which was 20 bases more, and the $\mathrm{XL}_{\mathrm{G}}$ was at 338 instead of 318 , which was, again, 20 bases more. This sample also had two additional peaks from the promoter region at 141 and 163 bases. Samples in Figures $2 b$-e were all heterozygous for the promoter ins/del polymorphism and $L$ peak of all the samples were more than 450, and we called it short/extra long, designated as $\mathrm{S} / \mathrm{XL}$. As the reverse primer was same for the LPR region and the A/G SNP, if there was a change in size of the $L$ allele the same size change was seen in the $L_{A} / L_{G}$ if the insertion

Table 2 Fragment sizes of different alleles detected and genotype calls.

\begin{tabular}{|c|c|c|c|c|c|c|c|c|c|c|c|c|c|}
\hline \multirow[t]{3}{*}{ Samples } & \multicolumn{9}{|c|}{ Promotor } & \multicolumn{3}{|c|}{ Intron 2 VNTR } & \multirow[t]{3}{*}{ Genotype } \\
\hline & \multirow[t]{2}{*}{$L$} & \multirow[t]{2}{*}{$s$} & \multirow[t]{2}{*}{$X L$} & \multicolumn{6}{|c|}{$S N P A / G$} & \multirow[t]{2}{*}{ StIn2.9 } & \multirow[t]{2}{*}{ StIn2.10 } & \multirow[t]{2}{*}{ StIn2.12 } & \\
\hline & & & & $S_{A}$ & $S_{G}$ & $L_{A}$ & $L_{G}$ & $X L_{A}$ & $X L_{G}$ & & & & \\
\hline C126 & 450 & & & & & 327 & & & & & 265 & & $\mathrm{~L}_{\mathrm{A}} / \mathrm{L}_{\mathrm{A}} / \mathrm{StIn} 2.10$ \\
\hline A97 & 450 & & & & & 327 & 318 & & & & & 297 & $\mathrm{~L}_{\mathrm{A}} / \mathrm{L}_{\mathrm{G}} / \mathrm{St} \ln 2.12$ \\
\hline M09 & & 406 & & 284 & & & & & & & & 297 & $\mathrm{~S}_{\mathrm{A}} / \mathrm{S}_{\mathrm{A}} / \mathrm{StIn} 2.12$ \\
\hline $\mathrm{R} 15$ & 450 & 406 & & 284 & & 327 & & & & & & 297 & $\mathrm{~L}_{\mathrm{A}} / \mathrm{S}_{\mathrm{A}} / \mathrm{St} \ln 2.12$ \\
\hline Sample 1 & & 406 & 470 & 283 & & & & & 338 & 248 & & 298 & $\mathrm{XL}_{\mathrm{G}} / \mathrm{S}_{\mathrm{A}} / \mathrm{StIn} 2.9 / \mathrm{S} t \ln 2.12$ \\
\hline Sample 2 & & 406 & 494 & 284 & & & & 371 & & & & 298 & $\mathrm{XL}_{\mathrm{A}} / \mathrm{S}_{\mathrm{A}} / \mathrm{St} \ln 2.12$ \\
\hline Sample 3 & & 407 & 492 & 284 & & & & 369 & & & & 298 & $\mathrm{XL}_{\mathrm{A}} / \mathrm{S}_{\mathrm{A}} / \mathrm{StIn} 2.12$ \\
\hline Sample 4 & & 406 & 537 & 284 & & & & 413 & & & & 297 & $\mathrm{XL}_{\mathrm{A}} / \mathrm{S}_{\mathrm{A}} / \mathrm{St} \ln 2.12$ \\
\hline Sample 5 & & 406 & 623 & 284 & & & & 504 & & & 265 & & $\mathrm{XL}_{\mathrm{A}} / \mathrm{S}_{\mathrm{A}} / \mathrm{St} \ln 2.10$ \\
\hline
\end{tabular}

Abbreviation: VNTR, variable number tandem repeat. 


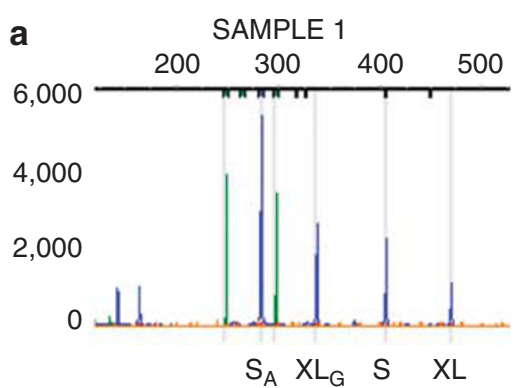

$9 \mathrm{R} \quad 12 \mathrm{R}$

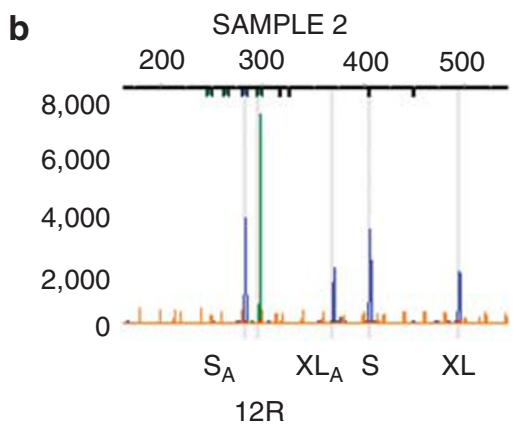

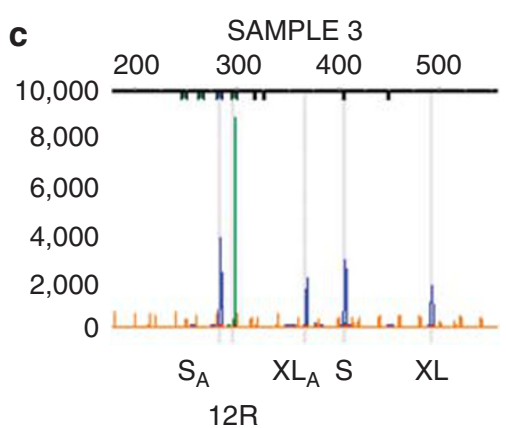

d

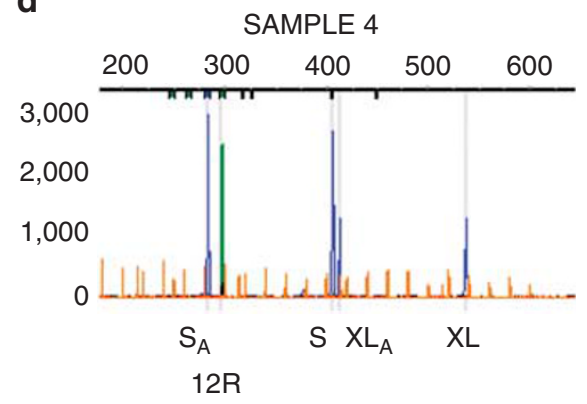

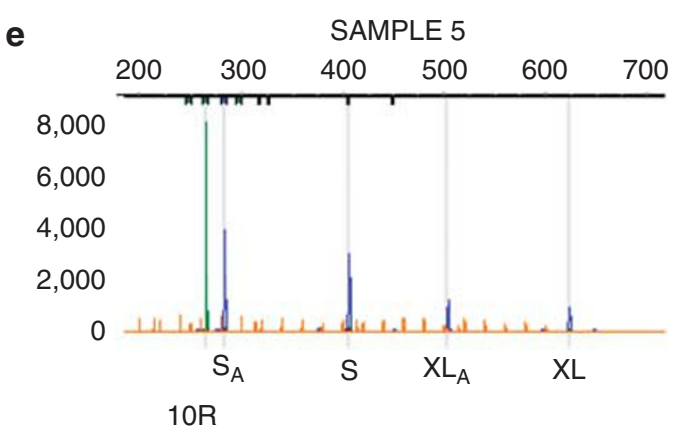

Figure 2 This figure shows the novel variants we found. In (a) the $\mathrm{XL}$ peak was at 470 instead of 450 and the $\mathrm{XL}_{\mathrm{G}}$ was at 338 , this sample had 17 repeats for the 5-HTTLPR XL allele. It was a StIn2.9/2.12. (b) Had an XL peak at 494 and the $X L_{A}$ peak was at 371, the XL allele had 18 repeats and this sample had Stln2.12 repeats. (c) Had an $X L$ peak at 492 and $X_{L_{A}}$ peak at 369, which was also an 18 repeat, this sample was Stln2.12. (d) Showed an XL peak at 537 and $X_{L_{A}}$ peak at 413 , the $X L$ allele was a 20-repeat unit and StIn2.12. (e) Showed an XL peak at 623 (approximate sizing because it was outside the ladder) and a $\mathrm{XL}_{\mathrm{A}}$ peak at 504, this was a 24-repeat unit and StIn2.10. All the five above samples were heterozygous for 5-HTTLPR and showed both $S$ and $S_{A}$ peak.

was after the A/G SNP. Sample 2 had an XL peak at 494 and the $X_{A}$ peak at 371 ; sample 3 had the $X L$ at 492 and $\mathrm{XL}_{\mathrm{A}}$ peak at 369; sample 4 had an $\mathrm{XL}$ peak at 537 and $\mathrm{XL}_{\mathrm{A}}$ peak at 413; and sample 5 had an $\mathrm{XL}$ peak at 623 (approximate because it was run with 600 base ladder) and the $\mathrm{XL}_{\mathrm{A}}$ peak at 504 .

Sequence verification of the novel alleles. The sequence data of the novel alleles are given in Supplementary information. We used the same Greek letter designation used by Nakamura et al..$^{5}$ for the different units.

The genetic architecture for the novel alleles are given in Figure 3 in comparison with $L_{A}$ and $L_{G}$. Sample 1 had the same units as the $L_{G}$ allele (16-D), $\alpha, \beta, \gamma, \delta, \varepsilon, \mu, o$, $\zeta, \eta, \theta, \imath, \kappa, \lambda, \mu, v, \xi$ until $\kappa$, and instead of $\lambda$ there was a $\zeta$ followed by two $\mu$ units instead of one $\mu$ (boldface in Figure 3); we are calling the first $\mu$ as $\mu^{\prime}$ because the sequence had an extra base $A$ at the end. This accounted for the extra 20 or 21 bases making this a 17-repeat unit. The two extra peaks at 141 and 163 bases were from the $\mu^{\prime}$ and $\mu$ units at the end.

Sample 2 and 3 had 18-repeat units. Sample 4 had 20-repeat units; these units were different from the 20-repeat unit reported before, ${ }^{5,6}$ which were $\alpha, \beta, \gamma, \delta, \varepsilon, \zeta, \eta, \zeta, \eta$, $\zeta, \eta, \zeta, \eta, \theta, \imath, \kappa, \lambda, \mu, v, \xi$. In sample 4 , the $\zeta$ unit (6th unit) was followed by $o, \zeta, \eta, \zeta, o, \zeta, \eta$; after $\eta$, it had the same pattern as $L_{A}$. Sample 5 was the longest one we found, with 24-repeat units (Figure 3 ).

\section{Discussion}

We developed a method for genotyping the following polymorphisms in human serotonin transporter: the 5-HTTLPR polymorphism, the rs25531 SNP in the promoter and the 
$L_{\mathrm{A}}(16 \mathrm{~A})$ \begin{tabular}{l|l|l|l|l|l|l|l|l|l|l|l|l|l|l|l|}
\hline$\alpha$ & $\beta$ & $\gamma$ & $\delta$ & $\varepsilon$ & $\zeta$ & $O$ & $\zeta$ & $\eta$ & $\theta$ & $\mathrm{T}$ & $\kappa$ & $\lambda$ & $\mu$ & $V$ & $\xi$ \\
\hline
\end{tabular} $\mathrm{L}_{\mathrm{G}}(16 \mathrm{D})$ \begin{tabular}{l|l|l|l|l|l|l|l|l|l|l|l|l|l|l|l|}
$\alpha$ & $\beta$ & $Y$ & $\delta$ & $\varepsilon$ & $\mu$ & 0 & $\zeta$ & $\eta$ & $\theta$ & $\mathrm{T}$ & $\mathrm{K}$ & $\lambda$ & $\mu$ & $\mathrm{V}$ & $\xi$ \\
\hline
\end{tabular}

Sample 1 XI 17 Repeat \begin{tabular}{l|l|l|l|l|l|l|l|l|l|l|l|l|l|l|l|l|}
\hline $\boldsymbol{\alpha}$ & $\boldsymbol{\beta}$ & $\mathrm{\gamma}$ & $\bar{\delta}$ & $\varepsilon$ & $\boldsymbol{\mu}$ & $\mathrm{o}$ & $\zeta$ & $\eta$ & $\theta$ & $\mathrm{r}$ & $\mathrm{K}$ & $\zeta$ & $\boldsymbol{\mu}$ & $\boldsymbol{\mu}$ & $\mathrm{v}$ & $\xi$ \\
\hline
\end{tabular}

Sample 2 XI 18 Repeat \begin{tabular}{l|l|l|l|l|l|l|l|l|l|l|l|l|l|l|l|l|l|}
\hline$\alpha$ & $\beta$ & $\gamma$ & $\delta$ & $\varepsilon$ & $\zeta$ & 0 & $\zeta$ & 0 & $\zeta$ & $\eta$ & $\theta$ & $\mathrm{T}$ & $\mathrm{K}$ & $\lambda$ & $\mu$ & $\mathrm{v}$ & $\xi$ \\
\hline
\end{tabular}

Sample 3 XI 18 Repeat \begin{tabular}{l}
$\alpha|\beta| \gamma|\delta|$ \\
\hline
\end{tabular}

Sample 4 XI 20 Repeat \begin{tabular}{l|l|l|l|l|l|l|l|l|l|l|l|l|l|l|l|l|l|l|l}
\hline $\boldsymbol{\alpha}$ & $\beta$ & $\gamma$ & $\delta$ & $\varepsilon$ & $\zeta$ & 0 & $\zeta$ & $n$ & $\zeta$ & 0 & $\zeta$ & $n$ & $\theta$ & $\mathrm{T}$ & $\mathrm{K}$ & $\boldsymbol{\lambda}$ & $\mu$ & $\mathrm{v}$ & $\xi$ \\
\hline
\end{tabular}

Sample 5 XI 24 Repeat

\begin{tabular}{l|l|l|l|l|l|l|l|l|l|l|l|l|l|l|l|l|l|l|l|l|l|l|l|}
\hline $\boldsymbol{\alpha}$ & $\beta$ & $\mathrm{r}$ & $\delta$ & $\varepsilon$ & $\zeta$ & 0 & $\zeta$ & 0 & $\zeta$ & $\eta$ & $\theta$ & $\mathrm{T}$ & $\mathrm{K}$ & $\lambda$ & $\mu$ & $\eta$ & $\theta$ & $\mathrm{T}$ & $\mathrm{K}$ & $\lambda$ & $\mu$ & $\mathrm{V}$ & $\xi$ \\
\hline
\end{tabular}

Figure 3 5-HTTLPR novel variants and the different units identified (the unique units are shown in boldface or within brackets).

StIn2. All four amplicons were amplified in separate reactions under the same cycling conditions, and the polymorphisms were detected simultaneously in a single electrophoretic step. This method eliminates time-consuming enzyme digestions and labor-intensive detection methods like running gels, and is more appropriate for a clinical setting. The analysis and interpretation is easy, and the entire assay is more costeffective. This method will be validated for routine clinical testing.

We identified novel variants in 5-HTTLPR using the method we developed, and the new variants were verified by direct DNA sequencing. We reported for the first time a 17-repeat allele (XL) with the SNP rs25531. This allele had the same units as $L_{G}$ until the $\kappa$ unit and differed by having a $\zeta$ instead of $\lambda$ followed by $\mu$ ' and $\mu$ units. The $\mu$ ' unit had the nucleotide $A$ at the end of the sequence. Typically the only unit that has an $A$ at the end is the $\lambda$ unit, which was missing at this position; therefore, this new repeat sequence must be a recombinant of $\mu$ and $\lambda$. The 17 repeat reported recently had a tandem duplication of a single $\kappa$ unit. $^{8}$ The $\mu$, which is the fourteenth unit in $L_{G}(16 D)$, is not usually picked up by the assay because the unit before that is $\lambda$, and the primer only anneals in this region weakly, whereas in this particular sample, as the unit before the $\mu$ ' was $\zeta$, the primer bound strongly and was detected.

The other four alleles we identified, the two 18 repeats, the 20 repeat and the 24 repeat followed the similar pattern, where the repeat elements were inserted after the $\zeta$ unit of $L_{A}$ (seventh unit). Of the two 18 repeats we detected, one had alternating units of $o$ and $\zeta$ from repeat 7 to 10 , which was identical to the one reported by Michaelovsky. ${ }^{7}$ The other 18 repeat had alternating units of $\eta$ and $\zeta$ from 7 to 10 repeat (the tandem repeats are shown in boldface in Figure 3 ), repeats 1 to 6 and 11 to18 were same in both alleles.

The 20-repeat allele we found had different units from the ones reported by Kunugi ${ }^{6}$ and Nakamura ${ }^{5}$ (boldface units in Figure 3) from repeat 7 to 11 . The 24-repeat allele had alternating units of $O$ and $\zeta$ from 7 to 10 repeat; units 11 to 16 and units 17 to 23 were in tandem repeat.
The two $\mu$ units (sixteenth and twenty-second unit) in the 24-repeat allele were not picked up by the assay as it was preceded by $\lambda$ units.

All the rare alleles we found were heterozygous samples with the $S$ allele and $X L$ allele as reported before. ${ }^{6}$ All the repeat insertions occurred after the $\zeta$ unit and seemed to be the hot spot for recombination. Also, the recombination region in all the variants were followed by the $\eta$ unit, and sometimes the $\eta$ unit is inserted repeatedly; this aligns with Heils et al.'s ${ }^{17}$ suggestion that presence of a sequence of 'hot spot' for deletion mutagenesis (TGCAGCC) is in the $\eta$ repeat element.

The 5-HTTLPR region is highly polymorphic, and repeats up to 24 units do exist in humans and are found to be a common occurrence in non-human primates. ${ }^{18}$ Presence of 5-HTTLPR in humans and simians but not in other mammals like mice may be related to anxiety-related personality traits so common in humans. ${ }^{18}$ The allelic distribution of the 5-HTT gene has remarkable ethnic variation, and the $\mathrm{XL}$ alleles seem to be largely present in the African or African-American population. Studies showed that $L$ and $X L$ alleles were more frequently found in the Japanese population in sudden infant death syndrome victims, chronic fatigue syndrome and temporomandibular disorder, ${ }^{19-21}$ whereas Haas et al. ${ }^{22}$ were not able to replicate the association between 5-HTT and sudden infant death syndrome in the Caucasian population. A large Taiwanese population displayed a much higher frequency of $\mathrm{XL}$ alleles than other studies, challenging the contention that the $\mathrm{XL}$ alleles are rare. ${ }^{23}$ As the $L$ allele shows more efficient serotonin function than the $S$ allele, this raises the question of whether the $\mathrm{XL}$ allele would exhibit even greater resilience to depression than the $L$ allele. ${ }^{23}$

Very few functional studies have been reported on these rare alleles. It is well established that the $L$ allele is associated with better and faster response to selective serotonin reuptake inhibitor therapy. Smeraldi et al. ${ }^{24}$ found significant differences in response to fluvoxamine among carriers of different alleles identified by Nakamura et al. ${ }^{5}$ They found that carriers of $16-\mathrm{F}$ showed only a partial response, whereas 16-D showed a marginally better response than 16-A allele carriers. The authors attribute this difference to the interaction of various transcription factors with the different consensus regions. ${ }^{24} A$ similar observation was made where transcription factors YB-1 ( $Y$ box-binding protein) and CTCF (CCTCbinding factor) have an important role in the regulation of the StIn2 polymorphism. ${ }^{25}$ Ehli et al. ${ }^{8}$ showed that there was no significant difference in expression between the extra short 11 repeat, and the $S$, and $L_{G}$ alleles, and a slight decrease of expression with the 17-repeat allele compared with $\mathrm{L}_{\mathrm{A}}$. It will be interesting to see if there is any difference in expression with the SNP rs25531 in the 17 repeat.

Serotonin uptake is genetically controlled, and dysregulation of 5-HTT function has been reported in various complex behavioral traits and disorders such as depression, bipolar disorder, anxiety, obsessive-compulsive disorder, schizophrenic and neurodegenerative disorders, substance abuse and eating disorders. ${ }^{26-31} 5$-HTT is the main target for widely used antidepressants, so it will be beneficial in the future to study the significance of these $\mathrm{XL}$ and other rare 
variants in correlation with selective serotonin reuptake inhibitor therapeutics.

\section{Conflict of interest}

The authors declare no conflict of interest.

1. Serretti A, Zanardi R, Franchini L, Artioli P, Dotoli D, Pirovano A et al. Pharmacogenetics of selective serotonin reuptake inhibitor response: a 6-month follow-up. Pharmacogenetics 2004; 14: 607-613

2. Serretti A, Kato M, De Ronchi D, Kinoshita T. Meta-analysis of serotonin transporter gene promoter polymorphism (5-HTTLPR) association with selective serotonin reuptake inhibitor efficacy in depressed patients. Mol Psychiatry 2007; 12: 247-257.

3. Hu XZ, Lipsky RH, Zhu G, Akhtar LA, Taubman J, Greenberg BD et al. Serotonin transporter promoter gain-of-function genotypes are linked to obsessive-compulsive disorder. Am J Hum Genet 2006; 78: 815-826.

4. Mrazek DA, Rush AJ, Biernacka JM, O'Kane DJ, Cunningham JM, Wieben ED et al SLC6A4 variation and citalopram response. Am J Med Genet B Neuropsychiatr Genet 2009; 150B: 341-351.

5. Nakamura M, Ueno S, Sano A, Tanabe H. The human serotonin transporter gene linked polymorphism (5-HTTLPR) shows ten novel allelic variants. Mol Psychiatry 2000; 5 32-38.

6. Kunugi H, Hattori M, Kato T, Tatsumi M, Sakai T, Sasaki T et al. Serotonin transporter gene polymorphisms: ethnic difference and possible association with bipolar affective disorder. Mol Psychiatry 1997; 2: 457-462.

7. Michaelovsky E, Frisch A, Rockah R, Peleg L, Magal N, Shohat M et al. A novel allele in the promoter region of the human serotonin transporter gene. Mol Psychiatry 1999; 4: 97-99.

8. Ehli EA, Hu Y, Lengyel-Nelson T, Hudziak JJ, Davies GE. Identification and functional characterization of three novel alleles for the serotonin transporter-linked polymorphic region. Mol Psychiatry 2011; 16: 1-8.

9. Praschak-Rieder N, Kennedy J, Wilson AA, Hussey D, Boovariwala A, Willeit M et al. Nove 5-HTTLPR allele associates with higher serotonin transporter binding in putamen: a [(11)C DASB positron emission tomography study. Biol Psychiatry 2007; 62: 327-331.

10. Wendland JR, Martin BJ, Kruse MR, Lesch KP, Murphy DL. Simultaneous genotyping of four functional loci of human SLC6A4, with a reappraisal of 5-HTTLPR and rs25531. Mol Psychiatry 2006; 11: 224-226.

11. Hranilovic D, Stefulj J, Schwab S, Borrmann-Hassenbach M, Albus M, Jernej B et al. Serotonin transporter promoter and intron 2 polymorphisms: relationship between allelic variants and gene expression. Biol Psychiatry 2004; 55: 1090-1094.

12. Ogilvie AD, Battersby S, Bubb VJ, Fink G, Harmar AJ, Goodwim GM et al. Polymorphism in serotonin transporter gene associated with susceptibility to major depression. Lancet 1996; 347: 731-733.

13. Collier DA, Arranz MJ, Sham $P$, Battersby S, Vallada H, Gill $P$ et al. The serotonin transporter is a potential susceptibility factor for bipolar affective disorder. Neuroreport 1996; 7: 1675-1679.

14. Rees M, Norton N, Jones I, McCandless F, Scourfield J, Holmans P et al. Association studies of bipolar disorder at the human serotonin transporter gene (hSERT; $5 \mathrm{HTT}$ ). Mol Psychiatry 1997; 2: 398-402

15. Hranilovic D, Schwab SG, Jernej B, Knapp M, Lerer B, Albus M et al. Serotonin transporter gene and schizophrenia: evidence for association/linkage disequilibrium in families with affected siblings. Mol Psychiatry 2000; 5: 91-95.
16. Lovejoy EA, Scott AC, Fiskerstrand CE, Bubb VJ, Quinn JP. The serotonin transporte intronic VNTR enhancer correlated with a predisposition to affective disorders has distinct regulatory elements within the domain based on the primary DNA sequence of the repeat unit. Eur J Neurosci 2003; 17: 417-420.

17. Heils A, Teufel A, Petri S, Stober G, Riederer P, Bengel $D$ et al. Allelic variation of human serotonin transporter gene expression. J Neurochem 1996; 66: 2621-2624.

18. Lesch KP, Meyer J, Glatz K, Flugge G, Hinney A, Hebebrand J et al. The 5-HT transporter gene-linked polymorphic region (5-HTTLPR) in evolutionary perspective: alternative biallelic variation in rhesus monkeys. Rapid communication. J Neural Transm 1997; 104 1259-1266.

19. Narita N, Narita M, Takashima S, Nakayama M, Nagai T, Okado N. Serotonin transporter gene variation is a risk factor for sudden infant death syndrome in the Japanese population. Pediatrics 2001; 107: 690-692.

20. Narita M, Nishigami N, Narita N, Yamaguti K, Okado N, Watanabe $Y$ et al. Association between serotonin transporter gene polymorphism and chronic fatigue syndrome. Biochem Biophys Res Commun 2003; 311: 264-266.

21. Ojima K, Watanabe N, Narita N, Narita M. Temporomandibular disorder is associated with a serotonin transporter gene polymorphism in the Japanese population. Biopsychosoc Med 2007; 1 : 3.

22. Haas C, Braun J, Bar W, Bartsch C. No association of serotonin transporter gene variation with sudden infant death syndrome (SIDS) in Caucasians. Leg Med (Tokyo) 2009; 11(Suppl 1): S210-S212.

23. Goldman N, Glei DA, Lin YH, Weinstein M. The serotonin transporter polymorphism (5-HTTLPR): allelic variation and links with depressive symptoms. Depress Anxiety 2010; 27: 260-269.

24. Smeraldi E, Serretti A, Artioli P, Lorenzi C, Catalano M. Serotonin transporter genelinked polymorphic region: possible pharmacogenetic implications of rare variants. Psychiatr Genet 2006; 16: 153-158.

25. Klenova E, Scott AC, Roberts J, Shamsuddin S, Lovejoy EA, Bergmann S et al. YB-1 and CTCF differentially regulate the 5-HTT polymorphic intron 2 enhancer which predisposes to a variety of neurological disorders. J Neurosci 2004; 24: 5966-5973.

26. Meltzer HY, Arora RC, Baber R, Tricou BJ. Serotonin uptake in blood platelets of psychiatric patients. Arch Gen Psychiatry 1981; 38: 1322-1326.

27. Stanley M, Virgilio J, Gershon S. Tritiated imipramine binding sites are decreased in the frontal cortex of suicides. Science 1982; 216: 1337-1339.

28. Perry EK, Marshall EF, Blessed G, Tomlinson BE, Perry RH. Decreased imipramine binding in the brains of patients with depressive illness. Br J Psychiatry 1983; 142: 188-192.

29. Stahl SM, Woo DJ, Mefford IN, Berger PA, Ciaranello RD. Hyperserotonemia and platelet serotonin uptake and release in schizophrenia and affective disorders. Am J Psychiatry 1983; 140: 26-30.

30. Joyce JN, Shane A, Lexow N, Winokur A, Casanova MF, Kleinman JE. Serotonin uptake sites and serotonin receptors are altered in the limbic system of schizophrenics. Neuropsychopharmacology 1993; 8: 315-336.

31. Owens MJ, Nemeroff CB. Role of serotonin in the pathophysiology of depression: focus on the serotonin transporter. Clin Chem 1994; 40: 288-295.

Translational Psychiatry is an open-access journal published by Nature Publishing Group. This work is licensed under the Creative Commons Attribution-NoncommercialNo Derivative Works 3.0 Unported License. To view a copy of this license, visit http://creativecommons.org/licenses/by-nc-nd/3.0/

\section{Supplementary Information accompanies the paper on the Translational Psychiatry website (http://www.nature.com/tp)}

